\title{
THREE POSITIVE PERIODIC SOLUTIONS FOR DYNAMIC EQUATIONS WITH PIECEWISE CONSTANT ARGUMENT AND IMPULSE ON TIME SCALES*
}

\author{
YONGKUN LI and ERLIANG XU \\ Department of Mathematics, Yunnan University, Kunming, Yunnan 650091, P.R. China
}

(Received 5 January 2010; accepted 28 June 2010; first published online 8 December 2010)

\begin{abstract}
In this paper, by using the Leggett-Williams fixed point theorem, the existence of three positive periodic solutions for differential equations with piecewise constant argument and impulse on time scales is investigated. Some easily verifiable sufficient criteria are established. Finally, an example is given to illustrate the results.
\end{abstract}

2010 Mathematics Subject Classification. 34N05, 34K45, 34K13.

1. Introduction. Impulsive differential equations, which arise in physics, population dynamics, economics, etc., are important mathematical tools for a better understanding of many real-world models, we refer the reader to [1-5] and the references therein. The study of differential equations on time scales, which has been created in order to unify the study of differential and difference equations, is an area of mathematics that has recently gained a lot of attention, moreover, many results on this issue have been well documented in the monographs [6-8]. The study of differential equations with piecewise constant arguments (EPCA) was initiated by Aftabizadeh and Wiener [9]. They observed that the change of sign in the argument deviation leads not only to interesting periodic properties but also to complications in the asymptotic and oscillatory behaviour of solutions. Various qualitative behaviours of solutions for EPCA have been investigated by many authors (see e.g. Refs. [9-17]).

To the best of the authors' knowledge, there have been no results about the existence of multiple solutions of impulsive differential equations with piecewise constant arguments and parameters. In this paper, by using the Leggett-Williams multiple fixed point theorem, we shall consider the following equation on time scales:

$$
\left\{\begin{array}{l}
x^{\Delta}(t)=-A(t) x^{\sigma}(t)+\lambda f(t, x(t), x(\beta(t))), \quad t \in \mathbb{T}, \quad t \neq t_{k}, \\
\Delta x\left(t_{k}\right)=x\left(t_{k}^{+}\right)-x\left(t_{k}^{-}\right)=I_{k}\left(x\left(t_{k}\right)\right), t=t_{k}, \quad k \in \mathbb{Z},
\end{array}\right.
$$

where $A(t)=\operatorname{diag}\left[a_{1}(t), a_{2}(t), \ldots, a_{n}(t)\right], f=\left(f_{1}, f_{2}, \ldots, f_{n}\right)^{T}, \lambda>0$ is a positive parameter; $\beta(t)=t_{k-1}$, if $t_{k-1} \leq t<t_{k}, k \in \mathbb{N}, t \in \mathbb{T}$, $\mathbb{T}$ is an $\omega$-periodic time scale. For each interval $I$ of $\mathbb{R}$, we denote $I_{\mathbb{V}}=I \cap \mathbb{T}, x\left(t_{k}^{+}\right)$and $x\left(t_{k}^{-}\right)$represent the right and the left limits of $x\left(t_{k}\right)$ in the sense of time scales; in addition, if $t_{k}$ is right-scattered, then $x\left(t_{k}^{+}\right)=x\left(t_{k}\right)$, whereas, if $t_{k}$ is left-scattered, then $x\left(t_{k}^{-}\right)=x\left(t_{k}\right)$. There exists a positive

*This work is supported by the National Natural Sciences Foundation of Peoples Republic of China under Grant 10971183. 
integer $p$ such that $t_{k+p}=t_{k}+\omega, I_{k+p}=I_{k}, k \in \mathbb{Z}$. Without loss of generality, we also assume that $[0, \omega]_{\mathbb{T}} \cap\left\{t_{k}, k \in \mathbb{Z}\right\}=\left\{t_{1}, t_{2}, \ldots, t_{q}\right\}$.

Throughout this paper, we assume that

$\left(H_{1}\right) a_{i} \in C\left(\mathbb{T}, \mathbb{R}_{+}\right)$is $\omega$-periodic, $i=1,2, \ldots, n$;

$\left(H_{2}\right) f \in C\left(\mathbb{T} \times \mathbb{R}_{+}^{n} \times \mathbb{R}_{+}^{n}, \mathbb{R}_{+}^{n}\right)$ is $\omega$-periodic with respect to the first variable and $I_{k} \in C\left(\mathbb{R}_{+}^{n}, \mathbb{R}_{+}^{n}\right), k \in \mathbb{Z}$.

2. Preliminaries. In this section, we shall recall some definitions, and state some preliminary results.

Definition 2.1. A function $x(t)$ is a solution of (1.1) on $\mathbb{T}$ if:

(i) $x(t)$ is continuous on $\mathbb{T}$;

(ii) the derivative $x^{\Delta}(t)$ exists at each point $t \in \mathbb{T}$, with the possible exception of the points $t_{k-1}, k \in \mathbb{Z}$, where one-sided derivatives exist;

(iii) Equation (1.1) is satisfied on each interval $\left[t_{k-1}, t_{k}\right), k \in \mathbb{Z}$.

Definition 2.2. [7] A time scale $\mathbb{T}$ is an arbitrary nonempty closed subset of the real numbers $\mathbb{R}$. The forward and backward jump operators $\sigma, \rho: \mathbb{T} \rightarrow \mathbb{T}$ and the graininess $\mu: \mathbb{T} \rightarrow \mathbb{R}^{+}$are defined, respectively, by

$$
\sigma(t):=\inf \{s \in \mathbb{T}: s>t\}, \rho(t):=\sup \{s \in \mathbb{T}: s<t\} \text { and } \mu(t)=\sigma(t)-t .
$$

Definition 2.3. [7] For $x: \mathbb{T} \rightarrow \mathbb{R}$, then we define the delta derivative of $x(t), x^{\Delta}(t)$, to be the number (if it exists) with the property that for a given $\varepsilon>0$, there exists a neighbourhood $U$ of $t$ such that

$$
\left|[x(\sigma(t))-x(t)]-x^{\Delta}(t)[\sigma(t)-s]\right| \leq \varepsilon|\sigma(t)-s|
$$

for all $s \in U$.

Definition 2.4. [7] If $X^{\Delta}(t)=x(t)$, then we define the delta integral by

$$
\int_{a}^{t} x(s) \Delta s=X(t)-X(a) .
$$

DefinITION 2.5. [18] Let $\mathbb{T} \neq \mathbb{R}$ be a periodic time scale with period $p$. We say that the function $f: \mathbb{T} \rightarrow \mathbb{R}$ is periodic with period $\omega$ if there exists a natural number $n$ such that $\omega=n p, f(t+\omega)=f(t)$ for all $t \in \mathbb{T}$ and $\omega$ is the smallest number such that $f(t+\omega)=f(t)$.

If $\mathbb{T}=\mathbb{R}$, we say that $f$ is periodic with period $\omega>0$ if $\omega$ is the smallest positive number such that $f(t+\omega)=f(t)$ for all $t \in \mathbb{T}$.

Definition 2.6. [7] A function $p: \mathbb{T} \rightarrow \mathbb{R}$ is called regressive if $1+\mu(t) p(t) \neq 0$ for all $t \in \mathbb{T}^{k}$, where $\mu(t)=\sigma(t)-t$ is the graininess function. If $p$ is regressive and rightdense continuous function, then the generalized exponential function $e_{p}$ is defined by

$$
e_{p}(t, s)=\exp \left\{\int_{s}^{t} \xi_{\mu(\tau)}(p(\tau)) \Delta \tau\right\}
$$


for $s, t \in \mathbb{T}$, with the cylinder transformation given by

$$
\xi_{h}(z)= \begin{cases}\frac{\log (1+h z)}{h}, & \text { if } h>0 \\ z, & \text { if } h=0\end{cases}
$$

Let $p, q: \mathbb{T} \rightarrow \mathbb{R}$ be two regressive functions, we define

$$
p \oplus q:=p+q+\mu p q, \quad \ominus p:=-\frac{p}{1+\mu p}, \quad p \ominus q=p \oplus(\ominus q) .
$$

Then the generalized exponential function has the following properties.

LeMma 2.1. [7] Assume that $p, q: \mathbb{T} \rightarrow \mathbb{R}$ are two regressive functions, then

(i) $e_{0}(t, s) \equiv 1$ and $e_{p}(t, t) \equiv 1$;

(ii) $e_{p}(\sigma(t), s)=(1+\mu(t) p(t)) e_{p}(t, s)$;

(iii) $e_{p}(t, \sigma(s))=\frac{e_{p}(t, s)}{1+\mu(s) p(s)}$;

(iv) $\frac{1}{e_{p}(t, s)}=e_{\ominus p}(t, s)$;

(v) $e_{p}(t, s)=\frac{1}{e_{p}(s, t)}=e_{\ominus p}(s, t)$;

(vi) $e_{p}(t, s) e_{p}(s, r)=e_{p}(t, r)$.

LEMMA 2.2. The function $x(t)$ is an $\omega$-periodic solution of (1.1) if and only if $x(t)$ is an $\omega$-periodic solution of the following system:

$$
x(t)=\lambda \int_{t}^{t+\omega} G(t, s) f(s, x(s), x(\beta(s))) \Delta s+\sum_{k: t_{k} \in[t, t+\omega]} G\left(t, t_{k}\right) I_{k}\left(x\left(t_{k}\right)\right),
$$

where

$$
G(t, s)=\operatorname{diag}\left[G_{1}(t, s), G_{2}(t, s), \ldots, G_{n}(t, s)\right]
$$

and

$$
G_{i}(t, s)=\frac{e_{\ominus a_{i}}(t, s)}{e_{\ominus a_{i}}(0, \omega)-1}, s \in[t, t+\omega]_{\mathbb{T}}, i=1,2, \ldots, n .
$$

Proof. First, we prove the necessity. If $x(t)=\left(x_{1}(t), \ldots, x_{n}(t)\right)^{T}$ is a solution of system (1.1), then

$$
x_{i}^{\Delta}(t)+a_{i}(t) x_{i}^{\sigma}(t)=\lambda f_{i}(t, x(t), x(\beta(t))),
$$

for $t \neq t_{k}, \quad i=1, \ldots, n$. Multiplying both sides of (2.2) by $e_{a_{i}}(t, 0)$, we have

$$
\left[x_{i}(t) e_{a_{i}}(t, 0)\right]^{\Delta}=\lambda f_{i}(t, x(t), x(\beta(t))) e_{a_{i}}(t, 0),
$$

for $i=1,2, \ldots, n$. Integrating (2.3) step by step from $t$ to $t+\omega$, we get

$x_{i}(t)=\lambda \int_{t}^{t+\omega} \frac{e_{\ominus a_{i}}(t, s)}{e_{\ominus a_{i}}(0, \omega)-1} f_{i}(s, x(s), x(\beta(s))) \Delta s+\sum_{j ; t_{j} \in[t, t+\omega]} \frac{e_{\ominus a_{i}}\left(t, t_{k}\right)}{e_{\ominus a_{i}}(0, \omega)-1} I_{i k}\left(x\left(t_{k}\right)\right)$,

where $i=1,2, \ldots, n$, we find that $x(t)$ satisfies $(2.1)$. 
Second, we prove the sufficiency. Let $x(t)=\left(x_{1}(t), \ldots, x_{n}(t)\right)^{T}$ be a solution of system (2.1). If $t \neq t_{k}, k \in \mathbb{Z}, i=1,2, \ldots, n$, from (2.1), we have

$$
\begin{aligned}
x_{i}^{\Delta}(t)= & \lambda\left[G_{i}(t, t+\omega) f_{i}(t+\omega, x(t+\omega), x(\beta(t+\omega)))-G_{i}(t, t) f_{i}(t, x(t), x(\beta(t)))\right] \\
& -a_{i}(t) x_{i}^{\sigma}(t) \\
= & -a_{i}(t) x_{i}^{\sigma}(t)+\lambda f_{i}(t, x(t), x(\beta(t))) .
\end{aligned}
$$

If $t=t_{k}, k \in \mathbb{Z}, i=1,2, \ldots, n$, then by (2.1), we get

$$
\begin{aligned}
x_{i}\left(t_{k}^{+}\right)-x_{i}\left(t_{k}^{-}\right) & =\sum_{j: t_{j} \in\left[t_{k}^{+}, t_{k}^{+}+\omega\right)} G_{i}\left(t_{k}, t_{j}\right) I_{i j}\left(x\left(t_{j}\right)\right)-\sum_{j: t_{j} \in\left[t_{k}^{-}, t_{k}^{-}+\omega\right)} G_{i}\left(t_{k}, t_{j}\right) I_{i j}\left(x\left(t_{j}\right)\right) \\
& =G_{i}\left(t_{k}, t_{k}+\omega\right) I_{i k}\left(x\left(t_{k}\right)+\omega\right)-G_{i}\left(t_{k}, t_{k}\right) I_{i k}\left(x\left(t_{k}\right)\right) \\
& =I_{i k}\left(x\left(t_{k}\right)\right) .
\end{aligned}
$$

So we know that, $x(t)$ is also an $\omega$-periodic solution of (1.1). This completes the proof Lemma 2.2.

Let $\mathbb{X}$ be a Banach space and $K$ be a closed, nonempty subset of $\mathbb{X} . K$ is a cone provided:

(i) $\alpha u+\beta v \in K$ for all $u, v \in K$ and $\alpha, \beta \geq 0$;

(ii) $u,-u \in K$ imply $u=0$.

Define $K_{r}=\{x \in K \mid\|x\| \leq r\}$. Let $\alpha(x)$ denote the positive continuous concave functional on $K$, that is, $\alpha: K \rightarrow[0, \infty)$ is continuous and satisfies

$$
\alpha(\lambda x+(1-\lambda) y) \geq \lambda \alpha(x)+(1-\lambda) \alpha(y) \text { for all } x, y \in K, 0 \leq \lambda \leq 1
$$

and we denote the set $K(\alpha, a, b)=\{x \mid x \in K, a \leq \alpha(x),\|x\| \leq b\}$.

The following lemma cited from Ref. [18] is useful for the proof of our main results of this paper.

LemmA 2.3. [19] Let $K$ be a cone of the real Banach space $\mathbb{X}$ and $A: K_{c} \rightarrow K_{c}$ be a completely continuous operator, and suppose that there exists a concave positive functional $\alpha$ with $\alpha(x) \leq\|x\|(x \in K)$ and numbers $a, b, d$ with $0<d<a<b \leq c$, satisfying the following conditions:

(1) $\{x \in K(\alpha, a, b): \alpha(x)>a\} \neq \emptyset$ and $\alpha(A x)>a$ if $x \in K(\alpha, a, b)$;

(2) $\|A x\|<d$ if $x \in K_{d}$;

(3) $\alpha(A x)>a$ for all $x \in K(\alpha, a, c)$ with $\|A x\|>b$.

Then $A$ has at least three fixed points in $K_{c}$.

In order to apply Lemma 2.2 to system (1.1), consider the Banach space

$$
\mathbb{X}=\left\{x \mid x=\left(x_{1}(t), \ldots, x_{n}(t)\right)^{T} \in P C\left(\mathbb{T}, \mathbb{R}^{n}\right): x(t+\omega)=x(t), t \in \mathbb{T}\right\}
$$

with the norm defined by $\|x\|=\sum_{i=1}^{n}\left|x_{i}\right|_{0}$, where $\left|x_{i}\right|_{0}=\sup _{t \in[0, \omega]_{\mathbb{T}}}\left|x_{i}(t)\right|$,

$$
P C\left(\mathbb{T}, \mathbb{R}^{n}\right)=\left\{x: \mathbb{T} \rightarrow \mathbb{R}^{n}|x|_{\left(t_{k}, t_{k+1}\right)_{\mathbb{T}}} \in C\left(\left(t_{k}, t_{k+1}\right) \mathbb{\mathbb { }}, \mathbb{R}^{n}\right), \exists x\left(t_{k}^{-}\right)=x\left(\left(t_{k}\right), k \in \mathbb{Z}\right\} .\right.
$$

Define the cone $K$ in $\mathbb{X}$ by

$$
K=\left\{x=\left(x_{1}(t), \ldots, x_{n}(t)\right)^{T} \in \mathbb{X}: x_{i}(t) \geq \sigma\left|x_{i}\right|_{0}, \quad t \in[0, \omega]_{\mathbb{T}}, i=1,2, \ldots, n\right\},
$$


where

$$
0<\sigma=A / B<1
$$

and

$$
\begin{gathered}
A=\min _{1 \leq i \leq n}\left\{A_{i}\right\}, B=\max _{1 \leq i \leq n}\left\{B_{i}\right\}, \\
A_{i}:=\min \left\{G_{i}(t, s): 0 \leq t \leq s \leq \omega\right\}=\frac{1}{e_{\ominus a_{i}}(0, \omega)-1}>0, i=1,2, \ldots, n, \\
B_{i}:=\max \left\{G_{i}(t, s): 0 \leq t \leq s \leq \omega\right\}=\frac{e_{\ominus a_{i}}(0, \omega)}{e_{\ominus a_{i}}(0, \omega)-1}>0, i=1,2, \ldots, n .
\end{gathered}
$$

It is not difficult to verify that $K$ is a cone in $X$.

Let the map $\Phi$ be defined by

$$
(\Phi x)(t)=\lambda \int_{t}^{t+\omega} G(t, s) f(s, x(s), x(\beta(s))) \Delta s+\sum_{k: t_{k} \in[t, t+\omega]} G\left(t, t_{k}\right) I_{k}\left(x\left(t_{k}\right)\right),
$$

for $x \in K, t \in \mathbb{T}$, and

$$
(\Phi x)=\left(\Phi_{1} x, \Phi_{2} x, \ldots, \Phi_{n} x\right)^{T}
$$

LEMma 2.4. The mapping $\Phi$ maps $K$ into $K$, i.e., $\Phi K \subset K$.

Proof. For any $x \in K$, it is easy to see that $\Phi x \in P C\left(\mathbb{T}, \mathbb{R}^{n}\right)$. Since $x(\beta(t+\omega))=$ $x(\beta(t))$, in view of $(2.4)$, for $t \in \mathbb{T}, i=1,2, \ldots, n$, we obtain

$$
\begin{aligned}
\left(\Phi_{i} x\right)(t+\omega)= & \lambda \int_{t+\omega}^{t+2 \omega} G_{i}(t+\omega, s) f_{i}(s, x(s), x(\beta(s))) \Delta s+\sum_{k: t_{k} \in[t+\omega, t+2 \omega]} G_{i}\left(t+\omega, t_{k}\right) I_{i k}\left(x\left(t_{k}\right)\right) \\
= & \lambda \int_{t}^{t+\omega} G_{i}(t+\omega, u+\omega) f_{i}(u+\omega, x(u+\omega), x(\beta(u+\omega))) \Delta u \\
& +\sum_{k: t_{k} \in[t, t+\omega]} G_{i}\left(t+\omega, t_{k}+\omega\right) I_{i k}\left(x\left(t_{k}+\omega\right)\right) \\
= & \lambda \int_{t}^{t+\omega} G_{i}(t, u) f_{i}(u, x(u), x(\beta(u))) \Delta u+\sum_{k: t_{k} \in[t, t+\omega]} G_{i}\left(t, t_{k}\right) I_{i k}\left(x\left(t_{k}\right)\right) \\
= & \left(\Phi_{i} x\right)(t) .
\end{aligned}
$$

That is, $(\Phi x)(t+\omega)=(\Phi x)(t), t \in \mathbb{T}$. So $\Phi x \in \mathbb{X}$. For any $x \in K, i=1,2, \ldots, n$, we have

$$
\left|\Phi_{i} x\right|_{0} \leq B_{i} \lambda \int_{t}^{t+\omega} f_{i}(t, x(t), x(\beta(t))) \Delta s+B_{i} \sum_{k: t_{k} \in[t, t+\omega]} I_{i k}\left(x\left(t_{k}\right)\right)
$$

and

$$
\left(\Phi_{i} x\right)(t) \geq A_{i} \lambda \int_{t}^{t+\omega} f_{i}(t, x(t), x(\beta(t))) \Delta s+A_{i} \sum_{k: t_{k} \in[t, t+\omega]} I_{i k}\left(x\left(t_{k}\right)\right) .
$$


So we get

$$
\left(\Phi_{i} x\right)(t) \geq \frac{A}{B}\left|\Phi_{i} x\right|_{0}=\sigma\left|\Phi_{i} x\right|_{0} .
$$

Hence, $\Phi x \in K$. The proof of Lemma 2.4 is complete.

Since the method is similar to that in the literature [20], we omit the proof of the following lemma.

LEMMA 2.5. The operator $\Phi: K \rightarrow K$ is completely continuous.

For convenience in the following discussion, we introduce the following notations:

$$
\begin{gathered}
f^{0}=\lim _{\|u\| \rightarrow 0} \sup _{t \in[0, \omega]} \frac{\|f(t, u(t), u(\beta(t)))\|}{\|u\|}, I^{0}=\limsup _{\|u\| \rightarrow 0} \sum_{k=1}^{q} \frac{\left\|I_{k}(u)\right\|}{\|u\|}, \\
f^{\infty}=\lim _{\|u\| \rightarrow \infty} \sup _{t \in[0, \omega]} \frac{\|f(t, u(t), u(\beta(t)))\|}{\|u\|}, I^{\infty}=\limsup _{\|u\| \rightarrow \infty} \sum_{k=1}^{q} \frac{\left\|I_{k}(u)\right\|}{\|u\|}
\end{gathered}
$$

and for $b>0$, we define

$$
I_{(b)}=\min _{\sigma b \leq\|u\| \leq b} \sum_{k=1}^{q}\left\|I_{k}(u)\right\| .
$$

\section{Main result.}

Our main result of this paper is as follows:

THEOREM 3.1. Assume that $\left(H_{1}\right)-\left(H_{2}\right)$ hold, there exists a number $b>0$ such that the following conditions:

(i) $\|f(t, u, u(\beta(t)))\|+(A+1) I_{(b)}>\frac{1+A}{A}\|u\|$ for $\sigma b \leq\|u\| \leq b, t \in \mathbb{T}$;

(ii) $f^{0}+I^{0}<\frac{1}{B}, f^{\infty}+I^{\infty}<\frac{1}{B}$

hold. Then the system (1.1) has at least three positive $\omega$-periodic solutions for

$$
\frac{1}{(A+1) \omega}<\lambda<\frac{1}{\omega} \text {. }
$$

Proof. By the second inequality in (ii), one can find an $\varepsilon>0$ such that

$$
\frac{\frac{1}{B}-\left(f^{\infty}+I^{\infty}\right)}{2}>\varepsilon>0 .
$$

By the definitions of $f^{\infty}$ and $I^{\infty}$, there exists a $C_{0}>b$ such that

$$
\|f(s, u, u(\beta(t)))\| \leq\left(f^{\infty}+\varepsilon\right)\|u\|, \quad \sum_{k=1}^{q}\left\|I_{k}(u)\right\| \leq\left(I^{\infty}+\varepsilon\right)\|u\|,
$$

where $\|u\|>C_{0}$. 
Let $C_{1}=C_{0} / \sigma$, if $x \in K,\|x\|>C_{1}$, then $\|x\|>C_{0}$ and we have

$$
\begin{aligned}
\|\Phi x\| & =\sup _{t \in[0, \omega]_{\pi}} \sum_{i=1}^{n}\left|\left(\Phi_{i} x\right)(t)\right| \\
& \leq \sum_{i=1}^{n}\left[\lambda B \int_{t}^{t+\omega} f_{i}(s, x(s), x(\beta(s))) \Delta s+B \sum_{k: t_{k} \in[t, t+\omega]} I_{i k}\left(x\left(t_{k}\right)\right)\right] \\
& =\lambda B \int_{t}^{t+\omega}\|f(s, x(s), x(\beta(s)))\| \Delta s+B \sum_{k: t_{k} \in[t, t+\omega]}\left\|I_{k}\left(x\left(t_{k}\right)\right)\right\| \\
& \leq \lambda B \int_{t}^{t+\omega}\left(f^{\infty}+\varepsilon\right)\|x\| \Delta s+B\left(I^{\infty}+\varepsilon\right)\|x\| \\
& \leq\left(\lambda B\left(f^{\infty}+\varepsilon\right) \omega+B\left(I^{\infty}+\varepsilon\right)\right)\|x\| \\
& <\|x\| .
\end{aligned}
$$

Take $K_{C_{1}}=\left\{x \mid=x \in K,\|x\| \leq C_{1}\right\}$, then the set $K_{C_{1}}$ is a bounded set. Since $\Phi$ is completely continuous, $\Phi$ maps bounded sets into bounded sets and there exists a number $C_{2}$ such that

$$
\|\Phi x\| \leq C_{2} \text { for any } x \in K_{C_{1}} .
$$

If $C_{2} \leq C_{1}$, we deduce that $\Phi: K_{C_{1}} \rightarrow K_{C_{1}}$ is completely continuous. If $C_{1}<C_{2}$, by (3.1), we know that for any $x \in K_{C_{2}} \backslash K_{C_{1}},\|x\|>C_{1}$ and $\|\Phi x\|<\|x\|<C_{2}$ hold. Thus, $\Phi: K_{C_{2}} \rightarrow K_{C_{2}}$ is completely continuous. Now, take $c=\max \left\{C_{1}, C_{2}\right\}$, obviously $c>b$, then $\Phi: K_{c} \rightarrow K_{c}$ is completely continuous.

Take the positive continuous concave functional $\alpha(x)=\sum_{i=1}^{n} \min _{t \in[0, \omega]_{\pi}}\left|x_{i}(t)\right|$. First, we let $a=\sigma b$ and take $x_{i} \equiv \frac{a+b}{2}, x \in K(\alpha, a, b), \alpha(x)>a$, then the set $\{x \in$ $K(\alpha, a, b)\} \neq \emptyset$. By (i) if $x \in K(\alpha, a, b)$, then $\alpha(x) \geq a$, and we have

$$
\begin{aligned}
\alpha(\Phi x) & =\sum_{i=1}^{n} \min _{t \in[0, \omega]_{\pi}}\left\{\lambda \int_{t}^{t+\omega} G_{i}(t, s) f_{i}(s, x(s), x(\beta(s))) \Delta s+\sum_{k: t_{k} \in[t, t+\omega]} G_{i}\left(t, t_{k}\right) I_{i k}\left(x\left(t_{k}\right)\right)\right\} \\
& \geq \sum_{i=1}^{n} \min _{t \in[0, \omega]_{\pi}}\left\{\lambda A \int_{t}^{t+\omega} f_{i}(s, x(s), x(\beta(s))) \Delta s+A \sum_{k=1}^{q} I_{i k}\left(x\left(t_{k}\right)\right)\right\} \\
& \left.\geq \lambda A \int_{0}^{\omega}\|f(s, x(s), x(\beta(s)))\| \Delta s+A \sum_{k=1}^{q}\left\|I_{k}\left(x\left(t_{k}\right)\right)\right\|\right\} \\
& >\lambda A \omega\left(\frac{A+1}{A} \alpha(x)-(A+1) I_{(b)}\right)+A I_{(b)} \\
& >\alpha(x) \geq a .
\end{aligned}
$$

Hence condition (1) of Lemma 2.3 holds.

Secondly, by the first inequality of condition of (ii), one can find $\varepsilon>0$ such that

$$
\frac{\frac{1}{B}-\left(f^{0}+I^{0}\right)}{2}>\varepsilon>0
$$


and there exists a $0<d<a$ such that

$$
\|f(s, u, u(\beta(t)))\| \leq\left(f^{0}+\varepsilon\right)\|u\|, \quad \sum_{k=1}^{q}\left\|I_{k}(u)\right\| \leq\left(I^{0}+\varepsilon\right)\|u\|,
$$

where $0 \leq\|u\| \leq d$. If $x \in K_{d}=\{x \mid\|x\| \leq d\}$, we have

$$
\begin{aligned}
\|\Phi x\| & =\sup _{t \in[0, \omega]_{\pi}} \sum_{i=1}^{n}\left|\left(\Phi_{i} x\right)(t)\right| \\
& \leq \sum_{i=1}^{n}\left[\lambda B \int_{t}^{t+\omega} f_{i}(s, x(s), x(\beta(s))) \Delta s+B \sum_{k: t_{k} \in[t, t+\omega]} I_{i k}\left(x\left(t_{k}\right)\right)\right] \\
& =\lambda B \int_{t}^{t+\omega}\|f(s, x(s), x(\beta(s)))\| \Delta s+B \sum_{k: t_{k} \in[t, t+\omega]}\left\|I_{k}\left(x\left(t_{k}\right)\right)\right\| \\
& \leq \lambda B \int_{t}^{t+\omega}\left(f^{0}+\varepsilon\right)\|x\| \Delta s+B\left(I^{0}+\varepsilon\right)\|x\| \\
& \leq\left(\lambda B\left(f^{0}+\varepsilon\right) \omega+B\left(I^{0}+\varepsilon\right)\right)\|x\| \\
& <\|x\| \leq d .
\end{aligned}
$$

That is, condition (2) of Lemma 2.3 holds.

Finally, if $x \in K(\alpha, a, c)$ with $\|\Phi x\| \geq b$, by the definition of the cone $K$, we get

$$
\begin{aligned}
b<\|\Phi x\| & \leq \sum_{i=1}^{n}\left[\lambda B \int_{t}^{t+\omega} f_{i}(s, x(s), x(\beta(s))) \Delta s+B \sum_{k: t_{k} \in[t, t+\omega]} I_{i k}\left(x\left(t_{k}\right)\right)\right] \\
& =\lambda B \int_{0}^{\omega}\|f(s, x(s), x(\beta(s)))\| \Delta s+B \sum_{k: t_{k} \in[t, t+\omega]}\left\|I_{k}\left(x\left(t_{k}\right)\right)\right\|,
\end{aligned}
$$

which implies that

$$
\begin{aligned}
\alpha(\Phi x) & =\sum_{i=1}^{n} \min _{t \in[0, \omega]_{\top}}\left\{\lambda \int_{t}^{t+\omega} G_{i}(t, s) f_{i}(s, x(s), x(\beta(s))) \Delta s+\sum_{k: t_{k} \in[t, t+\omega]} G_{i}\left(t, t_{k}\right) I_{i k}\left(x\left(t_{k}\right)\right)\right\} \\
& \geq \sum_{i=1}^{n} \min _{t \in[0, \omega]_{\top}}\left\{\lambda A \int_{0}^{\omega} f_{i}(s, x(s), x(\beta(s))) \Delta s+A \sum_{k=1}^{q} I_{i k}\left(x\left(t_{k}\right)\right)\right\} \\
& \geq \lambda A \int_{0}^{\omega}\|f(s, x(s), x(\beta(s)))\| \Delta s+A \sum_{k=1}^{q}\left\|I_{k}\left(x\left(t_{k}\right)\right)\right\| \\
& \geq \sigma\|\Phi x\|>\sigma b=a .
\end{aligned}
$$

So the condition (3) of Lemma 2.3 holds. Therefore, by Lemma 2.3, we obtain that the operator $\Phi$ has at least three fixed points in $K_{c}$. The proof of Theorem 3.1 is complete.

COROLlaRY 3.1. Using the following

$$
\text { (ii ) } f^{0}=0, I^{0}=0, f^{\infty}=0, I^{\infty}=0
$$

instead of (ii) in Theorem 3.1, the conclusion of Theorem 3.1 remains true. 


\section{An example.}

Consider the following equation:

$$
\begin{cases}x^{\prime}(t)=-\frac{1}{2} x(t)+\lambda x^{2}(t) e^{-x(\beta(t))}, & t \neq t_{k}, \\ \Delta x\left(t_{k}\right)=0.1 x^{2}\left(t_{k}\right) e^{-2 x\left(t_{k}\right)}, & t=t_{k}=2 k, k \in \mathbb{Z},\end{cases}
$$

where $\lambda$ is a non-negative parameter. Clearly, $A>0, B>0$. According to Corollary $3.1,(4.1)$ has at least three positive periodic solutions.

\section{REFERENCES}

1. V. Lakshmikantham, D. D. Bainov and P. S. Simeonov, Theorey of Impulsive Differential Equations (World Scientific, Singapore, 1989).

2. A. M. Samoilenko and N. A. Perestyuk, Impulsive Differential Equations (World Scientific, Singapore, 1995).

3. S. T. Zavalishchin and A. N. Sesekin, Dynamic Impulse Systems. Theory and applications (Kluwer Academic Publishers Group, Dordrecht, 1997).

4. W. Zhang and M. Fan, Periodic in a generalized ecological competition system governed by impulsive differential equations with delays, J. Math. Comput. Model. 39 (2004), 479-493.

5. D. D. Bainov and P. S. Simeonov, Impulsive differential equations: Periodic solutions and applications, in: Pitman Monographs and Surveys in Pure and Applied Mathematics, vol. 66 (Longman Scientific, New York, 1993).

6. V. Lakshmikantham, S. Sivasundaram and B. Kaymarkcalan, Dynamic systems on measure chains (Kluwer Academic Publishers, Dordrecht, 1996).

7. M. Bohner and A. Peterson, Dynamic equations on time scales, An introduction with applications (Birkhauser, Boston, 2001).

8. M. Bohner and A. Peterson, Advances in dynamic equations on time scales (Birkhauser, Boston, 2003).

9. A. R. Aftabizadeh and J. Wiener, Oscillatory and periodic solutions of an equation alternately of retarded and advanced type, Appl. Anal. 23 (1986), 219-231.

10. K. L. Cooke and J. Wiener, An equation alternately of retarded and advanced type, Proc. Amer. Math. Soc. 99 (1987), 726-732.

11. M. U. Akhmet, Almost periodic solutions of differential equations with piecewise constant argument of generalized type, Nonlinear Anal. 2 (2008), 456-467.

12. H. X. Li, Almost periodic solutions of second-order neutral equations with piecewise constant arguments, Nonlinear Anal. 65 (2006), 1512-1520.

13. H. X. Li, Almost periodic weak solutions neutral delay-differential equations with piecewise constant argument, Nonlinear Anal. 64 (2006), 530-545.

14. M. U. Akhmet, Stability of differential equations with piecewise constant arguments of generalized type, Nonlinear Anal. 68 (2008), 794-803.

15. M. Pinto, Asymptotic equivalence of nonlinear and quasi linear differential equations with piecewise constant arguments, Math. Comput. Model. 49 (2009), 1750-1758.

16. M. U. Akhmet and E. Yilmaz, Impulsive Hopfield-type neural networks system with piecewise constant argument, Nonlinear Anal. Real World Appl. 11 (2010), 2584-2593.

17. H. Liang, M. Z. Liu and W. J. Lv, Stability of $\theta$-schemes in the numerical solution of a partial dirrerential equation with piecewise continuous arguments, Appl. Math. Lett. 23 (2010), 198-206.

18. E. R. Kaufmann and Y. N. Raffoul, Periodic solutions for a neutral nonlinear dynamical equation on a time scale, J. Math. Anal. Appl. 319 (2006), 315-325.

19. R. W. Leggett and L. R. Williams, Multiple positive fixed points of nonlinear operator on ordered Banach spaces, Indiana Univ. Math. J. 28 (1979), 673-688.

20. J. J. Nieto, Basic theory for nonresonance impulsive periodic problems of first order, J. Math. Anal. Appl. 205 (1997), 423-433. 